\title{
The Complex Relationship Between Serum Uric Acid, Endothelial Function and Small Vessel Remodeling in Humans
}

\author{
Stefano Masi ${ }^{1,2,3, * \mathbb{C}}$, Georgios Georgiopoulos ${ }^{4,5,+}$, , George Alexopoulos ${ }^{6}$, \\ Konstantinos Pateras ${ }^{7}{ }^{1}$, Javier Rosada ${ }^{8}$, Gino Seravalle ${ }^{9}{ }^{(1)}$, Carolina De Ciuceis ${ }^{10}$, \\ Stefano Taddei ${ }^{1}$, Claudio Borghi ${ }^{11}$, Guido Grassi ${ }^{12}$, Damiano Rizzoni ${ }^{10,13,+\mathbb{1} \text {, }}$ \\ Agostino Virdis $1,+\infty$, the Study Groups on the Uric Acid Right for heArt Health (URRAH) $\ddagger$ and \\ Micro-and Macro-circulation of the Italian Society of Hypertension (SIIA) $\ddagger$ \\ 1 Department of Clinical and Experimental Medicine, University of Pisa, 56124 Pisa, Italy; \\ stefano.taddei@med.unipi.it (S.T.); agostino.virdis@med.unipi.it (A.V.) \\ 2 National Centre for Cardiovascular Prevention and Outcomes, Institute of Cardiovascular Science, \\ University College London, London EC1A 4NP, UK \\ 3 Department of Twin Research \& Genetic Epidemiology, King's College London, London WC2R 2LS, UK \\ 4 School of Biomedical Engineering and Imaging Sciences, King's College London, London WC2R 2LS, UK; \\ georgiopoulosgeorgios@gmail.com \\ 5 Department of Clinical Therapeutics, National and Kapodistrian University of Athens, 10679 Athens, Greece \\ 6 Department of Statistics, Athens University of Economics and Business, 10434 Athens, Greece; \\ gs.alexopoulos@gmail.com \\ 7 Department of Biostatistics and Research Support, Julius Center for Health Sciences and Primary Care, \\ University Medical Center Utrecht, 3584 CG Utrecht, The Netherlands; kostas.pateras@gmail.com \\ 8 Fourth Unit of Internal Medicine, University Hospital of Pisa, 56124 Pisa, Italy; j.rosada@ao-pisa.toscana.it \\ 9 Cardiology Unit, Fondazione Istituto Auxologico Italiano, Ospedale S. Luca, IRCCS Istituto Auxologico \\ Italiano, 20149 Milan, Italy; g_seravalle@yahoo.com \\ 10 Department of Clinical and Experimental Sciences, University of Brescia, 25121 Brescia, Italy; \\ cdeciuceis@gmail.com (C.D.C.); Damiano.rizzoni@unibs.it (D.R.) \\ 11 Department of Medical and Surgical Science, Alma Mater Studiorum University of Bologna, 40126 Bologna, \\ Italy; claudio.borghi@unibo.it \\ 12 Clinica Medica, Deptartment of Health Science, University Milano-Bicocca, 20126 Milan, Italy; \\ guido.grassi@unimib.it \\ 13 Division of Medicine, Spedali Civili di Brescia, Montichiari Hospital, 25018 Brescia, Italy \\ * Correspondence: stefano.masi@unipi.it \\ + These authors equally contributed to the manuscript. \\ $\ddagger \quad$ Name and affiliations of the study group members are reported in the acknowledgements.
}

Received: 29 May 2020; Accepted: 24 June 2020; Published: 28 June 2020

check for updates

\begin{abstract}
Aims: The relationship between serum uric acid (SUA) and microvascular remodeling in humans remains largely unexplored. We assessed whether SUA provides additional information on the severity of microvascular remodeling than that obtained from the European Heart Score (HS), the patterns of microvascular remodeling associated with changes in SUA levels and the mediation by endothelial function and nitric oxide (NO) availability on this relationship. Methods: A total of 162 patients included in the microvascular dataset of the Italian Society of Hypertension with available information on SUA, media-to-lumen (M/L) ratio, media cross-sectional area (MCSA), endothelial function, $\mathrm{NO}$ availability and $\mathrm{HS}$ were included in the analysis. The top tertile of $\mathrm{M} / \mathrm{L}$ ratio and MCSA were used to define severe microvascular remodeling. Results: A U-shaped association was observed between SUA and both M/L ratio and MCSA. Adjustment for HS did not affect these associations. SUA was able to reclassify a significant number of subjects without, and with, severe M/L ratio and MCSA remodeling over the HS alone. The microvascular remodeling associated with SUA levels presented a predominant hypertrophic pattern. SUA was inversely associated with endothelial
\end{abstract}


function and NO availability. Structural equation modeling analysis controlling for the HS suggested that the association of SUA with M/L ratio and MCSA was mediated through changes in endothelial function and NO availability. Conclusions: The addition of SUA to the HS improves the identification of subjects with greater microvascular remodeling. The relationship between SUA and microvascular remodeling is mediated by endothelial function and NO availability.

Keywords: uric acid; microvascular; remodeling; endothelial function

\section{Introduction}

A number of large epidemiologic studies have demonstrated the predictive value of serum uric acid (SUA) on the risk of cardiovascular (CV) disease and mortality, independently from common CV risk factors [1-3]. Based on these findings, European Guidelines have included SUA among factors to be assessed for the CV risk stratification of patients with arterial hypertension [4].

Increased circulating levels of SUA are not only related to the risk of clinical events but also to more severe organ damage [5-7], suggesting that SUA might promote the whole evolution of CV remodeling, from its subclinical manifestations to its clinical complications. This is in keeping with the mechanisms by which SUA is thought to contribute to the evolution of CV disease that lies in increased levels of vascular wall oxidative stress and inflammation [8]. Given that microvascular remodeling is currently considered the first manifestation of $\mathrm{CV}$ damage and is associated with the risk of $\mathrm{CV}$ events [9-13], a potential impact of SUA on microvascular remodeling might explain its association with future clinical and subclinical manifestations of CV disease. However, while several experimental models of asymptomatic hyperuricemia have provided evidence of a potential impact of SUA on microvascular remodeling [14-17], the incremental prognostic role of SUA on top of established CV factors for the severity of microvascular disease in humans has not yet been established.

We recently documented that endothelial dysfunction and, more specifically, a reduced nitric oxide (NO) availability, are strongly and independently related to measures of microvascular remodeling [18]. This is likely related to the capacity of endothelial function and NO availability to inform on both the damaging signals of $\mathrm{CV}$ risk factors and the capacity of the subjects to respond to such injuries. Common final pathways by which CV risk factors might alter the endothelial function and NO availability is by promoting the production of reactive oxygen species (ROS) and inflammatory cytokines, alterations that are also detected in subjects with hyperuricemia [19]. Thus, the capacity of SUA to promote the evolution of subclinical microvascular damage might relate to its influence on endothelial function.

In this study, we used the large microvascular data set from the Italian Society of Hypertension including microvascular, SUA and cardiovascular risk factor data from more than 100 subjects to assess: (i) whether SUA can provide additional information on the severity of resistance artery remodeling than that obtained from traditional scores used to define the patient's cardiovascular risk, (ii) the predominant pattern of small vessel remodeling associated with different SUA levels, and (iii) whether the relationship between SUA and measures of resistance artery remodeling is mediated by endothelial dysfunction and reduced $\mathrm{NO}$ availability.

\section{Methods}

\subsection{Population}

Analyses were performed using the population of the microvascular data set of the Italian Society of Hypertension who had available information on resistance artery structure and SUA levels. The characteristics of the data set have been previously described and reported in the supplementary material [20]. A diagram reporting the attrition of the population included in the Italian Society 
of Hypertension dataset based on the availability of the variables used to address each research question of this study is reported in the Supplementary Figure S1. To address the first and second research question of this study, we used data collected from three research centers in Italy (Brescia, Pisa, and Milano), for a total of 162 subjects with available measures of media-to-lumen (M/L) ratio and SUA levels. Among these $52 \%$ of the participants had a diagnosis of arterial hypertension, which was made according to the clinical history of the patient and confirmed by a direct recording of blood pressure values $>140 / 90 \mathrm{mmHg}$ during the clinical visit or the use of antihypertensive medications. Furthermore, 70 out of the 162 participants with available M/L ratio and SUA also had measures of endothelial function, which were used to address the third research hypothesis. The microvascular data set of the Italian Society of Hypertension has already shown its ability to provide reliable information on the relationship of small resistance artery remodeling with levels of cardiovascular risk factor, endothelial function and NO availability in previous publications $[18,20]$. The studies that contributed to the data set were conformed to the principles outlined in the Declaration of Helsinki, they were approved by the local ethics committees in each center, and the informed written consent for subsequent analyses was obtained from each participant.

\subsection{Measurements}

Information on the severity of small resistance artery remodeling, endothelial dysfunction and reduced NO availability were obtained using micromyography, as previously described $[18,20]$. Briefly, following isolation from biopsies of subcutaneous tissue collected from the anterior abdominal wall or gluteal regions, small resistance arteries were mounted on a pressure or wire myograph to assess the $\mathrm{M} / \mathrm{L}$ ratio and media cross-sectional area. From the structural parameters the growth and remodeling indexes were also calculated to define the predominant pattern of remodeling. The primary measure used to assess vascular remodeling was the $\mathrm{M} / \mathrm{L}$ ratio, as this has been shown to predict the individual's cardiovascular outcome [12,13]. Details of the methods used to assess M/L ratio, growth index, and remodeling index are reported in the supplementary file. The distribution of participants with and without arterial hypertension was different among the group of subjects assessed with the wire ( $80 \%$ hypertensives) or the pressure ( $40 \%$ hypertensives) myography. In 70 subjects recruited in Pisa, the acquisition of the remodeling parameters was followed by the assessment of endothelial function and NO availability, as previously reported and described in the supplementary material.

Serum Uric Acid was measured according to standardized protocols. Age, blood pressure values, hypertension status, body mass index (BMI), total cholesterol, high-density lipoprotein cholesterol, triglycerides, blood fasting glucose, and serum creatinine were collected and, for each patient, were used to calculate the heart score (HS) using the online tool provided by the European Guidelines on Cardiovascular Disease Prevention [21]. In addition, the estimated glomerular filtration rate (eGFR) was calculated using the Cockcroft-Gault formula.

\subsection{Statistics}

Data are expressed as mean \pm standard deviation for normally distributed variables or median $(25-75 \%)$ for not normally distributed variables. The maximal percentage increments or decrements of lumen diameter from baseline obtained from the dose-response curves to acetylcholine was used as a measure of endothelial function for statistical analyses. Initially, linear regression models were used to test the association between SUA and parameters of microvascular remodeling (M/L ratio and media cross-sectional area (MCSA)), endothelial function and NO availability. Indices of microvascular remodeling were used as dependent variables in the regression analysis after natural log transformation to decrease skewness and extreme observations influence.

To capture the nonlinear relationship between SUA levels and M/L ratio, we employed fractional polynomial analysis and considered various degrees of polynomial transformations for SUA. The best fitting second degree polynomial was selected among power transformations from the set, where 0 denotes the log transformation. The best fitting second-degree fractional polynomial was compared 
against simpler transformations of SUA model using a deviance difference test. For both $\mathrm{M} / \mathrm{L}$ and MCSA, a second-degree fractional polynomial with a linear and a quadratic term of SUA presented the optimal fit to the observed data. The same approach of fractional polynomial regression was employed to explore the association between levels of SUA and parameters defining the prevalent remodeling patterns (i.e., growth and remodeling index); a second-degree fractional polynomial with a linear and a quadratic term of SUA was selected for this analysis as previously described after standardization of the growth index and remodeling index. Given that an impaired renal function could influence the relationship between SUA and microvascular remodeling, these analyses were repeated excluding subjects with eGFR $<60 \mathrm{~mL} / \mathrm{min}$.

The additive value of SUA over the HS for classifying the severity of resistance artery remodeling in terms of M/L ratio and MCSA was estimated by the continuous net reclassification improvement [22]. A dichotomous outcome (severe remodeling defined as being classified in the highest tertile of $\mathrm{M} / \mathrm{L}$ or MCSA versus lower tertiles) was used in the net reclassification improvement analysis.

Subsequently, we assessed the attenuation of the relationships between SUA and measures of microvascular remodeling after adjustment for the potential mediators (endothelial function or NO availability). Such an attenuation was considered suggestive of a mediating effect of endothelial measures on the association between SUA and M/L ratio or MCSA. To confirm the possible mediating effect of endothelial function or NO availability on the relationships between SUA and microvascular remodeling and its significance we estimated the average causal mediation effect (ACME) by running 1000 bootstrap resamples with replacement and used structural equation modelling (SEM). Estimated ACME, average direct effect (ADE) and total effect are provided as median and $95 \% \mathrm{CI}$ after bootstrapping. All SEM model parameters were estimated by the full information maximum likelihood estimation (FIML) method, with respect to the missing data. We employed maximum likelihood estimation with "robust" (Huber-White) standard errors, and a robust test statistic (Satorra-Bentler chi-square test) for model evaluation to tackle deviation from normality in variables inserted in the SEM models. To evaluate the overall goodness of fit for the structural equation models, we used the model chi-square statistic test, and the root mean square error of approximation with values lesser or equal to 0.1 indicating good fit. Structural equation models were visualized with path diagrams. Statistical analyses were performed using Stata ver. 13.1 (StataCorp, College Station, TX, USA); the modules " $\mathrm{fp}$ " and " $\mathrm{mfp}$ " were used for univariable and multivariable fractional polynomial analysis, respectively. The lavaan package was used in $R$ ver. 3.5.3 to implement the SEM models. All statistical tests were two-tailed; we set the level of statistical significance at $p<0.05$.

\section{Results}

The characteristics of the 162 study participants with available SUA levels are reported in Table 1, stratified by tertiles of SUA. Prevalence of male gender, levels of triglycerides, ML, MCSA, and HS increased across ascending tertiles of SUA, while NO and acetylcholine (Ach) decreased (Table 1). 
Table 1. Characteristics of the population stratified by tertiles of serum uric acid (SUA).

\begin{tabular}{|c|c|c|c|c|c|}
\hline Variable & All (SD) & $\begin{array}{l}\text { 1st Tertile of } \\
\text { SUA }\end{array}$ & $\begin{array}{c}\text { 2nd Tertile of } \\
\text { SUA }\end{array}$ & $\begin{array}{l}\text { 3rd Tertile of } \\
\text { SUA }\end{array}$ & $p$-Value \\
\hline $\mathbf{N}$ & 162 & 57 & 53 & 52 & \\
\hline Age (years) & $46(14)$ & $45(14)$ & $45(14)$ & $51(13)$ & 0.067 \\
\hline Gender (male, \%) & $88(54)$ & $24(42)$ & $26(49)$ & $38(73)$ & 0.003 \\
\hline Smoking (never, \%) & $34(26)$ & $10(20)$ & $13(35)$ & $11(25)$ & 0.401 \\
\hline Serum Uric Acid (mg/dl) & $6.1(1.82)$ & $4.7(1.1)$ & $6.1(0.5)$ & $7.3(0.95)$ & $<0.001$ \\
\hline Systolic blood pressure (mmHg) & $142(18)$ & $139(17)$ & $144(21)$ & $142(16)$ & 0.456 \\
\hline Diastolic blood pressure $(\mathrm{mmHg})$ & $88(11)$ & $86(11)$ & $89(12)$ & $89(11)$ & 0.228 \\
\hline HDL-Cholesterol (mg/dl) & $47(10)$ & $49(11)$ & $48(10)$ & $44(9)$ & 0.06 \\
\hline LDL-Cholesterol (mg/dl) & $122(25)$ & $113(24)$ & $124(26)$ & $126(25)$ & 0.078 \\
\hline Glucose (mg/dl) & $97(24)$ & $97(26)$ & $97(26)$ & $97(19)$ & 0.989 \\
\hline Creatinine $(\mathrm{mg} / \mathrm{dl}) *$ & $0.88(0.34)$ & $0.83(0.23)$ & $0.96(0.32)$ & $0.87(0.35)$ & 0.004 \\
\hline eGFR $(\mathrm{ml} / \mathrm{min}){ }^{*}$ & $105(63.7)$ & $120(72)$ & $94.6(28.5)$ & $105(118)$ & 0.011 \\
\hline Diagnosis of Dyslipidemia (\%) & $58(53)$ & $17(41)$ & $15(56)$ & $26(63)$ & 0.132 \\
\hline Diagnosis of Hypertension (\%) & $84(52)$ & $29(51)$ & $27(51)$ & $28(54)$ & 0.941 \\
\hline Diagnosis of diabetes mellitus, $n(\%)$ & $19(12)$ & $11(20)$ & $5(10)$ & $3(6)$ & 0.072 \\
\hline $\mathrm{M} / \mathrm{L}$ ratio * & $0.079(0.042)$ & $0.082(0.042)$ & $0.063(0.036)$ & $0.084(0.04)$ & 0.008 \\
\hline $\operatorname{MCSA}\left(\mu \mathrm{m}^{2}\right)^{*}$ & $12,000(11,364)$ & $12,000(12,000)$ & $10,000(7200)$ & $13,000(12,000)$ & 0.019 \\
\hline Lumen $(\mu \mathrm{m})$ & $220(52)$ & $235(60)$ & $220(46)$ & $215(37)$ & 0.021 \\
\hline Lumen (pressurized myography) $(\mu \mathrm{m})$ & $218(39)$ & $221(42.6)$ & $215.5(27)$ & $217.1(48)$ & \\
\hline Lumen (wire myography) $(\mu \mathrm{m})$ & $250(91)$ & $266.41(111.71)$ & $252.05(76.49)$ & $174.8(37.88)$ & \\
\hline $\operatorname{Media}(\mu \mathrm{m})$ & $16.7(10.9)$ & $17.5(12.5)$ & $13.9(9.1)$ & $19.5(11,6)$ & 0.007 \\
\hline Media (pressurized myography) $(\mu \mathrm{m})$ & $14.5(9.45)$ & $13.4(7,5)$ & $13.2(2.2)$ & $18.72(11.24)$ & \\
\hline Media (wire myography) $(\mu \mathrm{m})$ & $21.5(8.24)$ & $28.89(8.0)$ & $23.15(6.79)$ & $19.93(11.28)$ & \\
\hline Remodeling index * & $999(2409)$ & $987(2100)$ & $1100(1000)$ & $929(2800)$ & 0.834 \\
\hline Growth Index ** & $30.9(12.13)$ & $30.9(12.1)$ & $30.9(12.1)$ & $36(26.2)$ & 0.521 \\
\hline Endothelial function $(\%) *$ & $74.5(15)$ & $76(19)$ & $75.3(18.5)$ & $72(6.8)$ & 0.035 \\
\hline NO Availability ** & $15(13.3)$ & 17 (11.5) & $15.4(14.8)$ & $13(6)$ & 0.026 \\
\hline Heart Score ${ }^{* *}$ & $0(1)$ & $0(1)$ & 0 (1) & $1(2)$ & 0.0025 \\
\hline
\end{tabular}

* Median and interquartile range. Abbreviations: HDL, high-density lipoprotein; LDL, low-density lipoprotein; eGFR, estimated glomerular filtration rate; and endothelial function, \% of maximal vasodilation compared to the baseline vascular diameter obtained with acetylcholine infusion. ${ }^{* *}$ Non-normally distributed variables.

\subsection{Relationship Between Uric Acid Levels and Microvascular Remodeling with and without Controlling for} Established Cardiovascular Risk Factors

We observed a U-shape association of SUA levels with $\mathrm{M} / \mathrm{L}$ ratio: low $(\beta=0.139$ unit change in $\log \mathrm{M} / \mathrm{L}$ ratio; $95 \%$ confidence interval $(\mathrm{CI})-0.001,0.280 ; p=0.051)$ and high tertile $(\beta=0.185$ unit change in $\log \mathrm{M} / \mathrm{L}$ ratio; $95 \% \mathrm{CI} 0.041,0.328 ; p=0.012$ ) of SUA, were related to increased $\mathrm{M} / \mathrm{L}$ ratio as compared to the middle (reference) tertile. Fractional polynomial analysis confirmed the non-linear association of continuous SUA with $\mathrm{M} / \mathrm{L}$ ratio $(\beta=-0.354 ; 95 \% \mathrm{CI}-0.557,-0.152 ; p<0.001$ for the linear term and $\beta=0.031 ; 95 \%$ CI $0.014,0.047 ; p<0.001$ for the non-linear, squared term) (Figure 1A). Similarly, SUA levels showed that a non-linear association with MCSA: low ( $\beta=0.142$ unit change in $\log \mathrm{M} / \mathrm{L}$ ratio; $95 \% \mathrm{CI}-0.033,0.317 ; p=0.112)$ and high tertile ( $\beta=0.246$ unit change in $\log \mathrm{M} / \mathrm{L}$ ratio; $95 \%$ CI $0.067,0.426 ; p=0.007$ ) of SUA, were related to increased MCSA as compared to the middle (reference) tertile. Fractional polynomial analysis confirmed the non-linear association of continuous SUA with MCSA ( $\beta=-1.59 ; 95 \% \mathrm{CI}-2.22,-0.952 ; p<0.001$ for the linear term and $\beta=0.581 ; 95 \% \mathrm{CI}$ $0.351,0.81 ; p<0.001$ for the non-linear, squared term) (Table 2) (Figure 1B). 

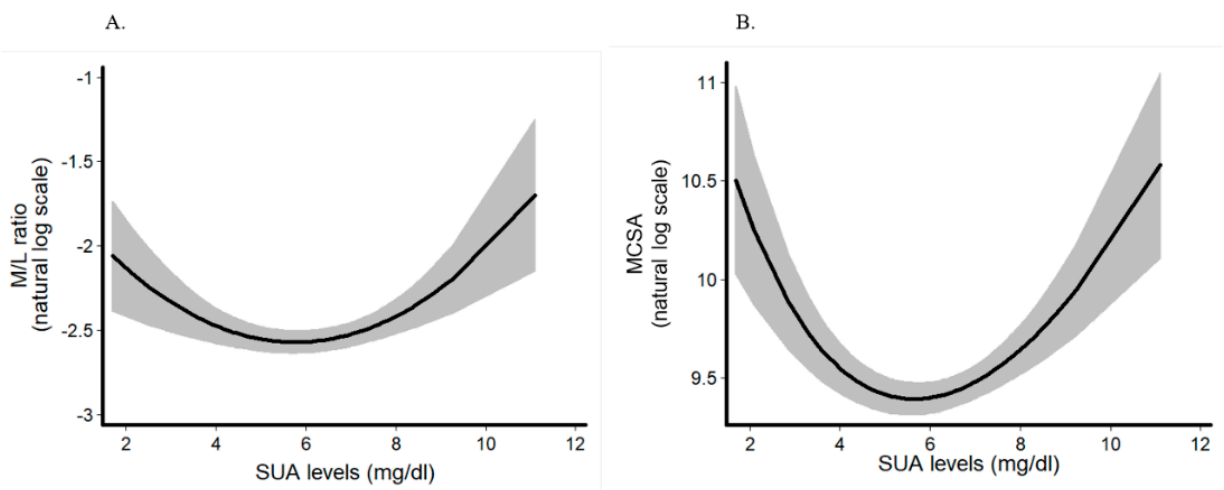

Figure 1. Fractional polynomial regression analysis for the association between continuous levels of serum uric acid (SUA) and (A) media-to-lumen ratio (M/L ratio) ( $n=162$ subjects) and (B) media cross-sectional area (MCSA) ( $n=157$ subjects). Serum uric acid is modelled as a second-degree fractional polynomial. A second-degree fractional polynomial with a linear and a quadratic term of SUA presented the optimal model fit to describe the relationship between SUA levels and remodeling parameters.

Table 2. Change of the relationship between SUA and parameters of vascular remodeling following adjustment for endothelial function and nitric oxide (NO) availability.

\begin{tabular}{|c|c|c|c|c|c|c|}
\hline & \multicolumn{2}{|c|}{ Unadjusted } & \multicolumn{2}{|c|}{$\begin{array}{l}\text { Adjusted for Endothelial } \\
\text { Function }\end{array}$} & \multicolumn{2}{|c|}{$\begin{array}{c}\text { Adjusted for NO } \\
\text { Availability }\end{array}$} \\
\hline & $\begin{array}{l}\text { Coefficient } \\
(95 \% \text { CI })\end{array}$ & $p$-Value & $\begin{array}{l}\text { Coefficient } \\
(95 \% \text { CI })\end{array}$ & $p$-Value & $\begin{array}{l}\text { Coefficient } \\
\text { (95\% CI) }\end{array}$ & $p$-Value \\
\hline \multicolumn{7}{|c|}{$\begin{array}{c}\text { Dependent } \\
\text { variable: } M / L \text { ratio }\end{array}$} \\
\hline SUA & $\begin{array}{c}-0.354 \\
(-0.557,-0.152) \\
\end{array}$ & $<0.001$ & $\begin{array}{c}-0.017 \\
(-0.034,0.067)\end{array}$ & 0.511 & $\begin{array}{c}0.012 \\
(-0.04,0.063)\end{array}$ & 0.647 \\
\hline \multicolumn{7}{|c|}{$\begin{array}{c}\text { Dependent } \\
\text { variable: MCSA }\end{array}$} \\
\hline SUA & $\begin{array}{c}-1.59 \\
(-2.22,-0.952)\end{array}$ & $<0.001$ & $\begin{array}{c}-0.015 \\
(-0.097,0.067)\end{array}$ & 0.716 & $\begin{array}{c}-0.027 \\
(-0.113,0.06)\end{array}$ & 0.541 \\
\hline
\end{tabular}

Abbreviations: $\mathrm{M} / \mathrm{L}$ ratio = media-to-lumen ratio; MCSA = media cross-sectional area; SUA = serum uric acid. Fractional polynomials were used to model the association of continuous uric acid with $\mathrm{M} / \mathrm{L}$ ratio and MCSA. Coefficients correspond to first degree polynomials or the linear term of second-degree polynomials. $\mathrm{M} / \mathrm{L}$ ratio and MCSA were used in the natural log transformed scale. Adjusted models additionally controlled for Heart Score.

The linear and non-linear associations between SUA and parameters of microvascular remodeling were significant in both sexes (Supplementary Table S1), in patients with and without hypertension (Supplementary Table S2) and exclusion of patients with eGFR $<60 \mathrm{~mL} / \mathrm{min}$ did not affect the results (Supplementary Table S3). Furthermore, adjustment for the HS did not attenuate the significant relationship of SUA with M/L ratio and MCSA ( $p<0.001$ for both).

SUA levels showed a hyperbolic association with growth index (standardized $\beta=25.6, p=0.01$ for the linear term and standardized $\beta=-24.6, p=0.014$ for the non-linear, squared term) (Supplementary Figure S2A). An inverse parabolic association was found between SUA levels and the remodeling index (standardized $\beta=1.08, p=0.021$ for the linear term and standardized $\beta=-0.078, p=0.041$ for the non-linear, squared term) (Supplementary Figure S2B). Given that we found a significant difference in the growth index between wire and pressure-myography, the analysis on the prevalent pattern of remodeling associated with SUA was repeated stratifying the population based on the method used for the microvascular assessment. As reported in the Supplementary Table S4, also the stratified analysis documented a prevalent pattern of hypertrophic remodeling associated with SUA, growth given that the growth index remained highly significantly associated with SUA in both models, while remodeling index lost its significance. 
By reclassification analysis, SUA correctly reclassified $7.14 \%$ and $45.16 \%$ of subjects without and with severe remodeling using $\mathrm{M} / \mathrm{L}$ ratio over $\mathrm{HS}(\mathrm{NRI}=0.523, \mathrm{SE}=0.218, p=0.016$ ). Substantial reclassification improvement was obtained with MCSA as well. Indeed, the addition of SUA to the HS led to a correct reclassification of $25 \%$ and $47.27 \%$ of subjects without and with severe MCSA remodeling, respectively; thus, accounting for a total NRI of $0.727, \mathrm{SE}=0.23, p=0.002$.

\subsection{Relationship Between Uric Acid Levels and Microvascular Remodeling: the Effect of Endothelial Function}

SUA was also associated with endothelial function and NO availability, so that subjects with higher SUA had a reduced endothelial-dependent vasodilation $(\beta=-4.86$ unit change in max vasodilation to Ach per $\mathrm{mg} / \mathrm{dl}$ increase in SUA; 95\% CI -7.91, -1.80; $p=0.003$ ). An even stronger relationship was identified, as expected, between SUA and levels of NO availability $(\beta=-5.72$ unit change in NO availability per $\mathrm{mg} / \mathrm{dl}$ increase in SUA; 95\% CI $-8.44,-2.99 ; p<0.001$ ). These associations remained highly significant when the analyses were restricted to subjects with a normal eGFR (Supplementary Table S3). The addition of end othelial function as independent variable led to a substantial attenuation of the association between SUA and both parameters of microvascular remodeling (Table 2). Similar results were obtained when NO availability was used instead of endothelial function (Table 2).

\subsection{Structural Equation Modelling}

By SEM analysis and after controlling for the clustering of established cardiovascular risk factors (Heart Score), SUA was positively associated indirectly with $\mathrm{M} / \mathrm{L}$ ratio (ACME $=0.28 \mathrm{per} \mathrm{mg} / \mathrm{dl}$ increase in SUA; 95\% CI 0.14, 0.43; $p<0.001$ ) and MCSA (ACME = 0.35 per mg/dl increase in SUA; 95\% CI 0.22, $0.48 ; p<0.001$ ), through changes in endothelial function. A direct effect was not established (for $\mathrm{M} / \mathrm{L}$ ratio: $\mathrm{ADE}=0.08 ; 95 \% \mathrm{CI}-0.05,0.21 ; p=0.22$ and for $\mathrm{MCSA}: \mathrm{ADE}=0.01 ; 95 \% \mathrm{CI}-0.16,0.17 ; p=0.94)$ (Table 3, Figure 2A,B). Both SEM models presented satisfactory fit to data (RMSE $\leq 0.1$ and chi-squared test $p>0.05)$.

Table 3. Estimation of the average direct effect (ADE) of serum uric acid levels and average causal mediation effect (ACME) through endothelial function and nitric oxide (NO) availability on parameters of microvascular remodeling. The average causal mediation effect (ACME) is the indirect effect of SUA on the outcomes through the mediator variables after controlling for Heart Score effect. The average direct effect (ADE) represents the direct effect exclusively explained by SUA on the remodeling indices.

\begin{tabular}{|c|c|c|c|c|c|c|}
\hline $\begin{array}{c}\text { Dependent } \\
\text { Variable }\end{array}$ & Mediator & Metric & Estimate & 95\% CI Lower & 95\% CI Upper & $p$-Value \\
\hline \multirow{3}{*}{$\mathrm{M} / \mathrm{L}$ ratio } & \multirow{3}{*}{$\begin{array}{l}\text { Endothelial } \\
\text { Function } \\
\text { (Ach) }\end{array}$} & ACME & 0.28 & 0.14 & 0.43 & $<0.01$ \\
\hline & & ADE & 0.08 & -0.05 & 0.21 & 0.22 \\
\hline & & Total Effect & 0.37 & 0.21 & 0.52 & $<0.01$ \\
\hline \multirow{3}{*}{$\mathrm{M} / \mathrm{L}$ ratio } & \multirow{3}{*}{$\begin{array}{c}\mathrm{NO} \\
\text { Availability }\end{array}$} & ACME & 0.35 & 0.22 & 0.48 & $<0.01$ \\
\hline & & $\mathrm{ADE}$ & 0.02 & -0.13 & 0.17 & 0.80 \\
\hline & & Total Effect & 0.37 & 0.21 & 0.52 & $<0.01$ \\
\hline \multirow{3}{*}{ MCSA } & \multirow{3}{*}{$\begin{array}{l}\text { Endothelial } \\
\text { Function } \\
\text { (Ach) }\end{array}$} & ACME & 0.28 & 0.14 & 0.43 & $<0.01$ \\
\hline & & ADE & 0.01 & -0.16 & 0.17 & 0.94 \\
\hline & & Total Effect & 0.29 & 0.13 & 0.45 & $<0.01$ \\
\hline \multirow{3}{*}{ MCSA } & \multirow{3}{*}{$\begin{array}{c}\mathrm{NO} \\
\text { Availability }\end{array}$} & ACME & 0.34 & 0.20 & 0.48 & $<0.01$ \\
\hline & & ADE & -0.05 & -0.24 & 0.14 & 0.60 \\
\hline & & Total Effect & 0.29 & 0.14 & 0.45 & $<0.01$ \\
\hline
\end{tabular}

Unstandardized effects were computed after bootstrapping with 1000 replicates 
A.

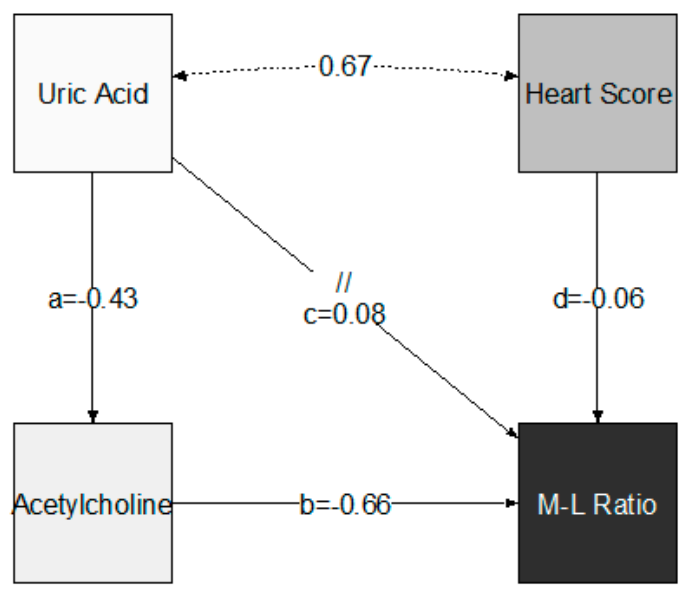

C.

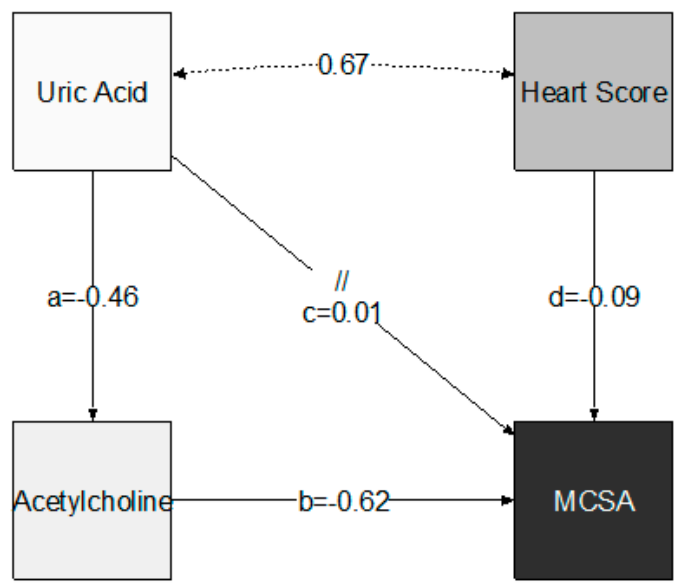

B.

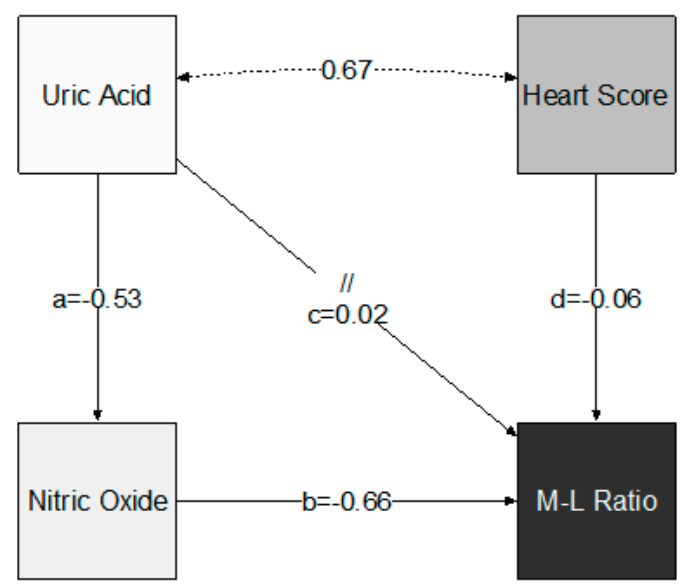

D.

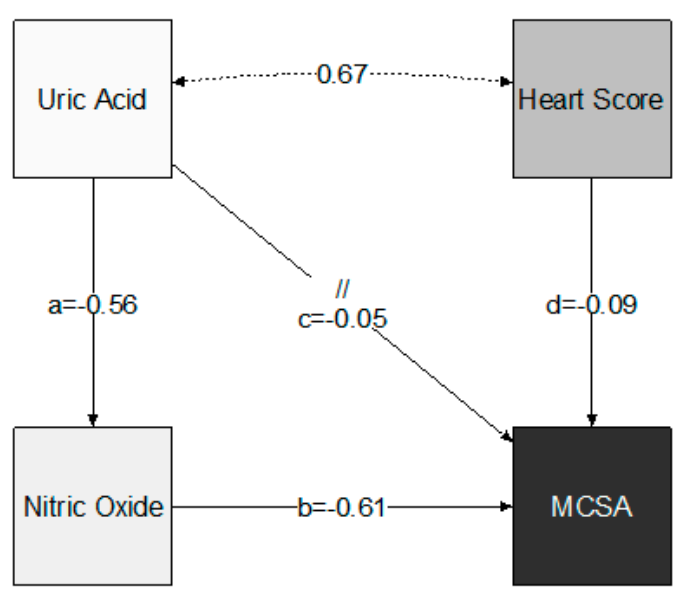

Figure 2. Path diagrams for structural equation models of the mediating effect of endothelial function ((A,C): Acetylcholine; (B,D): NO availability) on the association between uric acid levels and indices of microvascular remodeling (A and B: M/L ratio; $C$ and D: MCSA). Endothelial function was available in 70 subjects. In each path diagram, independent variables have unidirectional arrows pointing to the dependent variable. The parameter coefficient that has been estimated by the SEM models is placed adjacent to corresponding arrows. Coefficients $a$ and $b$ indicate the indirect effect $\left(a^{*} b\right)$ whilst coefficient c quantifies the direct effect. Double headed arrows represent the covariance estimation between uric acid levels and the Heart Score.

Accordingly, NO availability conferred significant mediation effect on the association of SUA with $\mathrm{M} / \mathrm{L}$ ratio (ACME $=0.35$ per $\mathrm{mg} / \mathrm{dl}$ increase in SUA; 95\% CI $0.22,0.48 ; p<0.001$ ) and MCSA $(\mathrm{ACME}=0.34$ per mg/dl increase in SUA; 95\% CI0.20, 0.48; $p<0.001$ ) (Table 3, Figure 2C,D). SEM analysis did not demonstrate significant direct effect of SUA on indices of microvascular remodeling (for M/L ratio: $\mathrm{ADE}=0.02 ; 95 \% \mathrm{CI}-0.13,0.17 ; p=0.80$ and for MCSA: $\mathrm{ADE}=-0.05 ; 95 \% \mathrm{CI}-0.24,0.14 ; p=0.60$ ) (Figure 2C,D). SEM fitting of the mediating effect of $\mathrm{NO}$ availability on $\mathrm{M} / \mathrm{L}$ ratio was satisfactory $(p=0.45)$ but suboptimal for MCSA $(p=0.023$, RMSE $=0.19)$.

\section{Discussion}

This is the first study to document a clear U-shaped association between SUA and parameters of microvascular remodeling in humans. Low and high levels of SUA were associated with hypertrophic remodeling of the small resistance vessels, characterized by higher $\mathrm{M} / \mathrm{L}$ ratio, MCSA, and growth 
index. The observed association was independent of other established CV risk factors, and the addition of SUA to the HS was able to reclassify a significant number of patients with severe and non-severe microvascular remodeling. Importantly, the effects of SUA on both M/L ratio and MCSA were mediated by endothelial function and NO availability. In summary, our results suggest that the measure of SUA might provide important additive information on the severity of microvascular remodeling than the HS alone. They also indicate that the assessment of endothelial function might represent an early and accurate marker to monitor the potential vascular benefits obtained with urate-lowering treatment.

An impact of SUA on microvascular remodeling has been described in animal models and cell culture experiments of hyperuricemia, where SUA can induce smooth muscle cell proliferation leading to increased MCSA and M/L ratio [14,16]. We now provide evidence that SUA levels is strongly associated with measures of small vessel remodeling also in humans and that the addition of SUA to the estimation of total CV risk with the use of the HS improves the capacity to identify patients with more or less severe small vessel remodeling. The U-shaped relationship between SUA and both $\mathrm{M} / \mathrm{L}$ ratio and MCSA observed in our study suggests that also very low levels of SUA might promote the evolution of microvascular disease. This is in keeping with the evidence that at physiological concentrations, SUA is a potent antioxidant in vitro $[23,24]$ and might have a protective effect on the vasculature. Therefore, low concentrations of SUA, particularly in extracellular fluids, could result in reduced antioxidant capacities, whilst high concentrations would promote its intracellular translocation, activating proliferative and pro-oxidant inflammatory pathways. The capacity of SUA to influence endothelial function due to its antioxidant effects is supported by in vivo studies. Indeed, uric acid administration improves endothelial function in the forearm vascular bed of patients with type 1 diabetes and in smokers. The extend of such improvements is similar to what can be obtained with the infusion of ascorbic acid, a potent antioxidant [25]. Further support on the capacity of uric acid to positively influence endothelial function is derived from patients affected by renal hypouricemia, a genetic disorder characterized by impaired reabsorption of uric acid and consequently low levels of SUA. Sugihara et al. reported that in patients with renal hypouricemia and SUA levels $<2.5 \mathrm{mg} / \mathrm{dl}$ endothelial function assessed by the flow-mediated dilation is correlated with SUA levels, and that patients with SUA $<0.8 \mathrm{mg} / \mathrm{dL}$ had a more pronounced impairment of the flow-mediated dilation than those with SUA between $0.8-2.5 \mathrm{mg} / \mathrm{dL}$ [26]. Compared to these reports, we now show that the negative effect of low levels of SUA on endothelial function might translate in higher vascular remodeling, potentially leading to an increased risk of cardiovascular disease. Indeed, several observational studies have documented a U- or J-shaped relationship between SUA and risk of CV events or mortality [27-33].

Although in vitro experiments have suggested that SUA might directly stimulate proliferation of smooth muscle cells $[16,34]$, our data suggests that on intact human small resistance arteries endothelial function and, more specifically, NO availability might play a key role in mediating the remodeling signal triggered by changes in the SUA levels. In cultured human endothelial cells, high concentrations of uric acid promote its translocation within the intracellular space, reducing NO availability by increasing ROS production [19] and through direct and irreversible reaction with the NO resulting in the formation of 6-aminouracil [35]. This is accompanied by the activation of vascular remodeling systems, such as the renin-angiotensin axis, which can further promote microvascular remodeling [19]. It is therefore possible that in intact vessels the endothelium represents the primary signal transducer of a high SUA concentration in the bloodstream, representing the intermediate mediator of its signals. Although our data support full mediation by NO availability and endothelial function on the relationship between SUA and small resistance artery remodeling, they do not exclude that in diseased conditions characterized by loss of endothelial integrity (i.e., in the context of atherosclerotic plaques) elevated concentrations of SUA might contribute to vascular remodeling by direct effects on other components of the vascular wall. Indeed, uric acid crystals have been detected within the vascular wall of atherosclerotic vessels [36]. Another potential explanation of our findings might lie in the capacity of SUA to mark the hyperactivity of the xanthine oxidase, the enzyme catalyzing the final reactions of the uric acid production, whom activity is also coupled with the production of ROS. 
Following this hypothesis, subjects with elevated SUA levels might represent those with an overactivity of the xanthine oxidase, leading also to increased production of ROS, endothelial dysfunction and, through this pathway, greater microvascular remodeling. Beyond the potential biological mechanisms underlying the relationship between SUA, microvascular remodeling, and endothelial function, our results support the use of endothelial function as an early indicator of the potential cardiovascular benefits achievable with urate-lowering treatments.

The low and high levels of SUA that show the strongest association with the M/L ratio are related to a predominant pattern of hypertrophic remodeling, with a prevalent association with the growth rather than the remodeling index. The association of high SUA levels with hypertrophic remodeling is in keeping with previous animal and cultured experiments, showing that SUA can promote smooth muscle cell proliferation leading to hypertrophy of the media microvascular layer $[14,16]$. Conversely, the strong association evidenced in our study between low SUA levels and hypertrophic remodeling has not been described previously in humans. We can only speculate on the biological mechanisms accounting for this association. For example, the lack of adequate antioxidant defenses in subjects with low SUA levels might result in a deficit of NO availability which, in turn, has been associated with a prevalent pattern of hypertrophic rather than eutrophic remodeling [18].

The microvascular dataset of the Italian Society of Hypertension has some unique features for the study of the relationship between parameters of vascular remodeling, endothelial function, and CV risk factors. The large sample size for a microvascular study and availability of data on a wide range of established risk factors and potential confounders enabled the independent role of many parameters to be assessed. The micromyography is currently considered the gold standard technique to assess resistance artery structural changes, and the database of the Italian Society of Hypertension has already proven its reliability for analyses assessing the complex relationships between $\mathrm{CV}$ disease risk factors and parameters of microvascular remodeling $[18,20]$. The $\mathrm{M} / \mathrm{L}$ ratio, used as primary endpoint of our analysis, is a validated measure of microvascular remodeling and has been shown to predict cardiovascular events in populations at high and low cardiovascular risk $[12,13]$. Previous publications have shown a substantial overlap of the $\mathrm{M} / \mathrm{L}$ ratio obtained with the pressure and wire micromyography [37]. In addition, its measure is far less influenced by possible sampling bias, an issue that could affect other parameters of microvascular structure, such as the lumen diameter and the media. Finally, the availability of information on endothelial function and NO availability in a large subsample of participants enabled a proper mediation analysis to be conducted, largely enhancing our understanding of the mechanisms underpinning the relationship between SUA, endothelial dysfunction, and vascular remodeling.

Our work also has important limitations. The observational and cross-sectional study design do not provide the opportunity to assign/infer causality from our data reliably. However, it should be emphasized that longitudinal studies using the micromyography technique are limited by the availability of repeated collection of biopsy samples due to ethical constraints. Nevertheless, despite having cross-sectional data, we were able to conduct a formal mediation analysis to identify the direction of the associations between SUA, remodeling parameters, endothelial function, and NO availability. We did not have information on measures of vascular wall oxidative stress, making any explanation on the mechanisms potentially accounting for the endothelial dysfunction in participants with hyperuricemia speculative. The report lacks hard cardiovascular endpoints. However, the scope of our study was not to confirm the relationship between SUA and CV outcomes, rather it was aimed at identifying potential mechanisms underpinning this associations and the measure of $\mathrm{M} / \mathrm{L}$ ratio has been previously proven to be a potent predictor of $\mathrm{CV}$ endpoints, independently from the levels of common CV risk factors. Given that the microvascular dataset of the Italian Society of Hypertension was established by merging data from studies conducted in order to answer different research questions, some important information potentially influencing the relationship between SUA and microvascular remodeling were missing. Among these, we did not have information on the prior diagnosis of gout, making it impossible to assess whether the impact of hyperuricemia on parameters 
of microvascular remodeling/function differs among subjects with or without grout. Moreover, we did not have information on treatments; thus, we could not assess the effect of urate lowering drugs, or antihypertensive drugs, on the relationship between SUA and microvascular remodeling parameters.

In conclusion, this study suggests that the addition of SUA to the assessment of common CV risk factors might significantly improve the capacity to identify people at increased risk of microvascular remodeling in the general population. Given that small vessel disease is considered the earliest organ damage related to CV risk factor exposure, this relationship might explain the associations between SUA and CV events described in several large observational studies. In humans without atherosclerosis, the influence of SUA on the vascular phenotype is likely mediated by its impact on endothelial function and NO availability.

Supplementary Materials: The following are available online at http://www.mdpi.com/2077-0383/9/7/2027/s1, Methods; Table S1. The linear and non-linear relationship between SUA and parameters of microvascular remodeling in males and females, Table S2. The linear and non-linear relationship between SUA and parameters of microvascular remodeling in subjects with and without arterial hypertension, Table S3. The linear and non-linear relationship between SUA and parameters of microvascular remodeling/endothelial function in subjects with eGFR $>60 \mathrm{~mL} / \mathrm{min}$, Table S4. Differences in the relationship of growth and remodeling indexes among microvascular assessment methods, Figure S1. Diagram of the attrition of the population included in the SIIA adaset based on the availability of data for each analysis. Abbreviations: HS = Heart Score, Figure S2. Fractional polynomial regression analysis for the association between continuous levels of serum uric acid (SUA) and (A) growth index $(n=118)$ and $(\mathrm{B})$ remodeling index $(n=72)$. Serum uric acid is modelled as a second-degree fractional polynomial.

Author Contributions: S.M., S.T., C.B., G.G., D.R., A.V. generated the research hypothesis, contributed to the analyses and critically revised the manuscript. G.G. (Georgios Georgiopoulos), G.A., K.P. performed the analyses. J.R., G.S., C.D.C., G.G. (Guido Grassi), D.R., A.V. acquired the data. S.M., A.V., D.R., S.T., G.G. (Guido Grassi) drafted the manuscript. All authors have read and agreed to the published version of the manuscript.

Funding: This work was supported by institutional fundings of the Department of Clinical and Experimental Medicine, University of Pisa, Italy obtained from the Ministero dell'Istruzione, dell'Università e della Ricerca, Italy.

Acknowledgments: We would like to acknowledge Emiliano Duranti who has provided substantial support in creating the Microvascular Dataset of the Italian Society of Hypertension. URRAH Project participating centers and investigators: Arrigo F.G. Cicero from the Department of Medical and Surgical Science, Alma Mater Studiorum University of Bologna, Bologna, Italy; Maria Lorenza Muiesan and Massimo Salvetti from the Department of Clinical and Experimental Sciences, University of Brescia, Italy; Andrea Ungar and Giulia Rivasi from the Department of Geric and Intensive Care Medicine, Careggi Hospital and University of Florence, Italy; Roberto Pontremoli and Francesca Viazzi from the Department of Internal Medicine, University of Genoa and Policlinico San Martino, Genova, Italy; Giovambattista Desideri, Claudio Ferri and Bruno Bernardino from the Department of Life, Health and Environmental Sciences, University of L'Aquila, L'Aquila, Italy; Luciano Lippa, from the Italian Society of General Medicine (SIMG), Avezzano, L'Aquila, Italy; Michele Bombelli and Rita Facchetti from Department of Medicine and Surgery, Clinica Medica, University of Milano-Bicocca, Italy; Gianfranco Parati from the Department of Cardiovascular, Neural and Metabolic Sciences, S. Luca Hospital, Istituto Auxologico Italiano and University of Milan-Bicocca, Milan, Italy; Ferruccio Galletti and Lanfranco D'Elia from the Department of Clinical Medicine and Surgery, "Federico II" University of Naples Medical School, Naples, Italy; Edoardo Casiglia and Paolo Palatini from the Studium Patavinum, Department of Medicine, University of Padua, Padua, Italy; Valerie Tikhonoff from the Department of Medicine, University of Padua, Padua, Italy; Carlo M. Barbagallo from the Biomedical Department of Internal Medicine and Specialists, University of Palermo, Palermo, Italy; Paolo Verdecchia from the Hospital S. Maria della Misericordia, Perugia, Italy; Francesca Mallamaci from the CNR-IFC, Clinical Epidemiology of Renal Diseases and Hypertension, Reggio Cal Unit, Reggio Calabria, Italy; Massimo Cirillo from the Department of Public Health, "Federico II" University of Naples, Napoli, Italy; Marcello Rattazzi from the Department of Medicine-DIMED, University of Padova, Medicina Interna $1^{\circ}, \mathrm{Ca}^{\prime}$ Foncello University Hospital, Treviso, Italy; Pietro Cirillo and Loreto Gesualdo from the Department of Emergency and Organ Transplantation-Nephrology, Dialysis and Transplantation Unit, Aldo Moro University of Bari, Bari, Italy; Alberto Mazza from the Department of Internal Medicine, Hypertension Unit, General Hospital, Rovigo, Italy; Cristina Giannattasio and Alessandro Maloberti from Cardiology IV, "A. De Gasperis" Department, Niguarda Ca' Granda Hospital, Health Science Department, Milano-Bicocca University, Milano, Italy; Massimo Volpe and Giuliano Tocci from the Hypertension Unit, Division of Cardiology, Department of Clinical and Molecular Medicine, Faculty of Medicine and Psychology, University of Rome Sapienza, Sant'Andrea Hospital, Rome, and IRCCS Neuromed, Pozzilli (IS), Italy; Guido Iaccarino from the Department of Advanced Biomedical Sciences, "Federico II" University of Naples, Napoli, Italy; and Pietro Nazzaro from the Department of Medical Basic Sciences, Neurosciences and Sense Organs, University of Bari Medical School, Bari, Italy.

Conflicts of Interest: The authors declare no conflict of interests. 


\section{References}

1. Clarson, L.E.; Chandratre, P.; Hider, S.L.; Belcher, J.; Heneghan, C.; Roddy, E.; Mallen, C.D. Increased cardiovascular mortality associated with gout: A systematic review and meta-analysis. Eur. J. Prev. Cardiol. 2015, 22, 335-343. [CrossRef]

2. Virdis, A.; Masi, S.; Casiglia, E.; Tikhonoff, V.; Cicero, A.F.; Ungar, A.; Rivasi, G.; Salvetti, M.; Barbagallo, C.M.; Dell'Oro, R.; et al. Identification of the Uric Acid Thresholds Predicting an Increased Total and Cardiovascular Mortality Over 20 Years. Hypertension 2020, 75, 302-308. [CrossRef]

3. Casiglia, E.; Tikhonoff, V.; Virdis, A.; Masi, S.; Barbagallo, C.M.; Bombelli, M.; Bruno, B.; Cicero, A.F.G.; Cirllo, M.; Cirllo, P.; et al. Serum uric acid and fatal myocardial infarction: Detection of prognostic cut-off values: The URRAH (Uric Acid Right for Heart Health) study. J. Hypertens. 2020, 38, 412-419. [CrossRef]

4. Williams, B.; Mancia, G.; Spiering, W.; Agabiti Rosei, E.; Azizi, M.; Burnier, M.; Clement, D.L.; Coca, A.; de Simone, G.; Kahan, T.; et al. 2018 ESC/ESH Guidelines for the management of arterial hypertension. Eur. Heart J. 2018, 39, 3021-3104. [CrossRef]

5. Lambert, E.A.; Hachem, M.; Hemmes, R.; Straznicky, N.E.; Eikelis, N.; Sari, C.I.; Schlaich, M.P.; Lambert, G.W.; Dixon, J.B. Serum uric acid and the relationship with subclinical organ damage in adults. J. Hypertens. 2017, 35, 745-752. [CrossRef]

6. Viazzi, F.; Parodi, D.; Leoncini, G.; Parodi, A.; Falqui, V.; Ratto, E.; Vetoretti, S.; Bezante, G.P.; Sette, M.D.; Pontremoli, R.; et al. Serum uric acid and target organ damage in primary hypertension. Hypertension 2005, 45, 991-996. [CrossRef]

7. Muiesan, M.L.; Agabiti-Rosei, C.; Paini, A.; Salvetti, M. Uric Acid and Cardiovascular Disease: An Update. Eur. Cardiol. 2016, 11, 54-59. [CrossRef]

8. Feig, D.I.; Kang, D.H.; Johnson, R.J. Uric acid and cardiovascular risk. New Engl. J. Med. 2008, 359, $1811-1821$. [CrossRef]

9. Buus, N.H.; Mathiassen, O.N.; Fenger-Grøn, M.; Præstholm, M.N.; Sihm, I.; Thybo, N.K.; Schroeder, A.P.; Thygesen, K.; Aalkjaer, C.; Mulvany, M.J.; et al. Small artery structure during antihypertensive therapy is an independent predictor of cardiovascular events in essential hypertension. J. Hypertens. 2013, 31, $791-797$. [CrossRef]

10. Heagerty, A.M. Predicting hypertension complications from small artery structure. J. Hypertens. 2007, 25, 939-940. [CrossRef]

11. Izzard, A.S.; Rizzoni, D.; Agabiti-Rosei, E.; Heagerty, A.M. Small artery structure and hypertension: Adaptive changes and target organ damage. J. Hypertens. 2005, 23, 247-250. [CrossRef] [PubMed]

12. Mathiassen, O.N.; Buus, N.H.; Sihm, I.; Thybo, N.K.; Mørn, B.; Schroeder, A.P.; Thygesen, K.; Aalkjaer, C.; Lederballe, O.; Christensen, K.L.; et al. Small artery structure is an independent predictor of cardiovascular events in essential hypertension. J. Hypertens. 2007, 25, 1021-1026. [CrossRef] [PubMed]

13. Rizzoni, D.; Porteri, E.; Boari, G.E.; De Ciuceis, C.; Sleiman, I.; Muiesan, M.L.; Castellano, M.; Miclini, M.; Agabiti-Rosei, E. Prognostic significance of small-artery structure in hypertension. Circulation 2003, 108, 2230-2235. [CrossRef]

14. Corry, D.B.; Eslami, P.; Yamamoto, K.; Nyby, M.D.; Makino, H.; Tuck, M.L. Uric acid stimulates vascular smooth muscle cell proliferation and oxidative stress via the vascular renin-angiotensin system. J. Hypertens. 2008, 26, 269-275. [CrossRef]

15. Kang, D.H.; Park, S.K.; Lee, I.K.; Johnson, R.J. Uric acid-induced C-reactive protein expression: Implication on cell proliferation and nitric oxide production of human vascular cells. J. Am. Soc. Nephrol. JASN 2005, 16, 3553-3562. [CrossRef]

16. Mazzali, M.; Hughes, J.; Kim, Y.G.; Jefferson, J.A.; Kang, D.H.; Gordon, K.L.; Lan, H.Y.; Kivlighn, S.; Johnson, R.J. Elevated uric acid increases blood pressure in the rat by a novel crystal-independent mechanism. Hypertension 2001, 38, 1101-1106. [CrossRef]

17. Rao, G.N.; Corson, M.A.; Berk, B.C. Uric acid stimulates vascular smooth muscle cell proliferation by increasing platelet-derived growth factor A-chain expression. J. Biol. Chem. 1991, 266, 8604-8608. [PubMed]

18. Masi, S.; Georgiopoulos, G.; Chiriacò, M.; Grassi, G.; Seravalle, G.; Savoia, C.; Volpe, M.; Taddei, S.; Virdis, A. The importance of endothelial dysfunction in resistance artery remodelling and cardiovascular risk. Cardiovasc. Res. 2020, 116, 429-437. [CrossRef] [PubMed] 
19. Yu, M.A.; Sanchez-Lozada, L.G.; Johnson, R.J.; Kang, D.H. Oxidative stress with an activation of the renin-anotensin system in human vascular endothelial cells as a novel mechanism of uric acid-induced endothelial dysfunction. J. Hyperten 2010, 28, 1234-1242.

20. Bruno, R.M.; Grassi, G.; Seravalle, G.; Savoia, C.; Rizzoni, D.; Virdis, A. Age- and Sex-Specific Reference Values for Media/Lumen Ratio in Small Arteries and Relationship With Risk Factors. Hypertension 2018, 71, 1193-1200. [CrossRef] [PubMed]

21. Piepoli, M.F.; Hoes, A.W.; Agewall, S.; Albus, C.; Brotons, C.; Catapano, A.L.; Cooney, M.-T.; Cosyns, B.; Deaton, C.; Graham, I.; et al. 2016 European Guidelines on cardiovascular disease prevention in clinical practice: The Sixth Joint Task Force of the European Society of Cardiology and Other Societies on Cardiovascular Disease Prevention in Clinical Practice (constituted by representatives of 10 societies and by invited experts)Developed with the special contribution of the European Association for Cardiovascular Prevention \& Rehabilitation (EACPR). Eur. Heart J. 2016, 37, 2315-2381. [PubMed]

22. Pencina, M.J.; D'Agostino, R.B., Sr.; Steyerberg, E.W. Extensions of net reclassification improvement calculations to measure usefulness of new biomarkers. Stat. Med. 2011, 30, 11-21. [CrossRef] [PubMed]

23. Ames, B.N.; Cathcart, R.; Schwiers, E.; Hochstein, P. Uric acid provides an antioxidant defense in humans against oxidant- and radical-caused aging and cancer: A hypothesis. Proc. Natl. Acad. Sci. USA 1981, 78, 6858-6862. [CrossRef]

24. Fabbrini, E.; Serafini, M.; Colic Baric, I.; Hazen, S.L.; Klein, S. Effect of plasma uric acid on antioxidant capacity, oxidative stress, and insulin sensitivity in obese subjects. Diabetes 2014, 63, 976-981. [CrossRef]

25. Waring, W.S.; McKnight, J.A.; Webb, D.J.; Maxwell, S.R. Uric acid restores endothelial function in patients with type 1 diabetes and regular smokers. Diabetes 2006, 55, 3127-3132. [CrossRef] [PubMed]

26. Sugihara, S.; Hisatome, I.; Kuwabara, M.; Niwa, K.; Maharani, N.; Kato, M.; Ogino, K.; Hamada, T.; Ninomiya, H.; Ichida, K.; et al. Depletion of Uric Acid Due to SLC22A12 (URAT1) Loss-of-Function Mutation Causes Endothelial Dysfunction in Hypouricemia. Circ. J. Off. J. Jpn. Circ. Soc. 2015, 79, 1125-1132. [CrossRef]

27. Cho, S.K.; Chang, Y.; Kim, I.; Ryu, S. U-Shaped Association Between Serum Uric Acid Level and risk of Mortality: A Cohort Study. Arthr. Rheumatol. 2018, 70, 1122-1132. [CrossRef]

28. Kuo, C.F.; See, L.C.; Yu, K.H.; Chou, I.J.; Chiou, M.J.; Luo, S.F. Significance of serum uric acid levels on the risk of all-cause and cardiovascular mortality. Rheumatology 2013, 52, 127-134. [CrossRef] [PubMed]

29. Tseng, W.C.; Chen, Y.T.; Ou, S.M.; Shih, C.J.; Tarng, D.C.; Taiwan Geriatric Kidney Disease Research Group. U-Shaped Association Between Serum Uric Acid Levels With Cardiovascular and All-Cause Mortality in the Elderly: The Role of Malnourishment. J. Am. Heart Assoc. 2018, 7, e007523. [CrossRef]

30. Culleton, B.F.; Larson, M.G.; Kannel, W.B.; Levy, D. Serum uric acid and risk for cardiovascular disease and death: The Framingham Heart Study. Ann. Intern. Med. 1999, 131, 7-13. [CrossRef]

31. Kamei, K.; Konta, T.; Hirayama, A.; Ichikawa, K.; Kubota, I.; Fujimoto, S.; Narita, I. Associations between serum uric acid levels and the incidence of nonfatal stroke: A nationwide community-based cohort study. Clin. Exp. Nephrol. 2017, 21, 497-503. [CrossRef]

32. Verdecchia, P.; Schillaci, G.; Reboldi, G.; Santeusanio, F.; Porcellati, C.; Brunetti, P. Relation between serum uric acid and risk of cardiovascular disease in essential hypertension. PIUMA Study Hypertens. 2000, 36, 1072-1078. [CrossRef]

33. Zhang, W.; Iso, H.; Murakami, Y.; Miura, K.; Nagai, M.; Sugiyama, D.; Ueshima, H.; Okamura, T.; EPOCH-JAPAN GROUP. Serum Uric Acid and Mortality Form Cardiovascular Disease: EPOCH-JAPAN Study. J. Atheroscler. Thromb. 2016, 23, 692-703. [CrossRef]

34. Kang, D.H.; Nakagawa, T.; Feng, L.; Watanabe, S.; Han, L.; Mazzali, M.; Truong, L.; Harris, R.; Johnson, R.J. A role for uric acid in the progression of renal disease. J. Am. Soc. Nephrol. JASN 2002, 13, 2888-2897. [CrossRef]

35. Gersch, C.; Palii, S.P.; Kim, K.M.; Angerhofer, A.; Johnson, R.J.; Henderson, G.N. Inactivation of nitric oxide by uric acid. Nucleosides Nucleotides Nucleic Acids 2008, 27, 967-978. [CrossRef] [PubMed] 
36. Patetsios, P.; Song, M.; Shutze, W.P.; Pappas, C.; Rodino, W.; Ramirez, J.A.; Panetta, T.F. Identification of uric acid and xanthine oxidase in atherosclerotic plaque. Am. J. Cardiol. 2001, 88, 188-191. [CrossRef]

37. Schiffrin, E.L.; Deng, L.Y. Structure and function of resistance arteries of hypertensive patients treated with a beta-blocker or a calcium channel antagonist. J. Hypertens. 1996, 14, 1247-1255. [CrossRef] [PubMed]

(C) 2020 by the authors. Licensee MDPI, Basel, Switzerland. This article is an open access article distributed under the terms and conditions of the Creative Commons Attribution (CC BY) license (http://creativecommons.org/licenses/by/4.0/). 